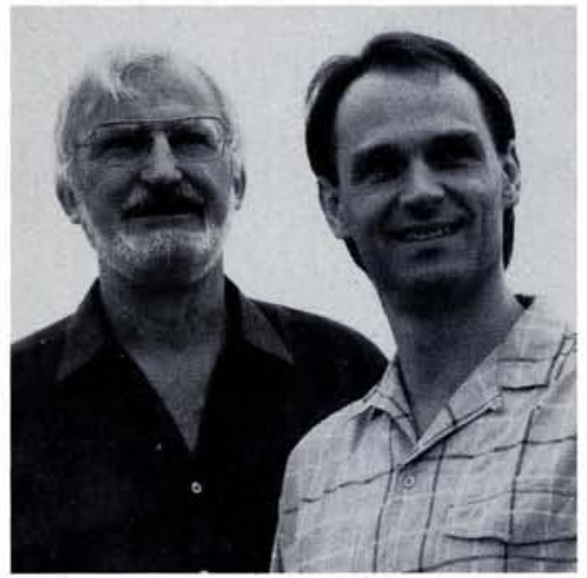

On October 15, 1986 the world learned about the award of the Nobel Prize in Physics to Ernst Ruska for his fundamental investigations in electron optics and the construction of the first electron microscope, and to Gerd Binnig and Heinrich Rohrer for designing the scanning tunnelling microscope.

Ruska, now 79, obtained satisfactory images with his two-stage instrument in 1931 and surpassed the resolution and magnification of light microscopes two years later while working at the Technical University in Berlin ${ }^{1}$ ). Since then electron microscopes with their different imaging modes and modifications, have slowly matured and gained acceptance as indispensable tools in physics, materials science, microelectronics, biology and medicine that enable structural analysis and selective manipulation on a scale of few $\mu \mathrm{m}$ down to a few $\AA$ (under special conditions). It is therefore gratifying that the Prize Committee decided to honour Ruska's pioneering work, thus recognizing what The New York Times referred to as a "microscopic oversight".

By contrast, the Scanning Tunnelling Microscope (STM), first successfully operated only five years ago, soon proved able to resolve atomic-scale features on the surfaces of even poorly conducting materials. Although it is only beginning to have an impact beyond fundamental science, the tremendous potential of STM (depending on the context, $M$ means either microscope or microscopy) has been realized by the Prize Committee at a relatively early stage.

\title{
1986 Nobel Prizes Development of Scanning Tunnelling Microscopy
}

\author{
A. Baratoff, Zürich
}

(IBM Zürich Research Laboratory)

Heinrich Rohrer (left) and Gerd Binnig (also winners of the 1984

EPS Hewlett-Packard Europhysics Prize).

Both instruments rely on electrons and are limited by quantum properties of the latter, but have otherwise little in common. The present contribution focusses on the development of STM by its inventors. A similar article on electron microscopy will appear later. Before reviewing the main stages in the evolution of the STM (see also Ref. 2), let me describe the physics and operation of that conceptually simple instrument. The state of the art, including complete references, is covered in two recent Proceedings ${ }^{3,4}$ ).

\section{Basic Principles}

The key to STM is to approach a probing tip, made of a refractory hard metal like tungsten, within a few $\AA$ of the sample to be investigated so that a measurable current $I_{T}$ (typically $1 \mathrm{nA}$ ) flows in response to a fixed voltage $V_{T}$ ranging from a few $\mathrm{mV}$ for metals to several volts for poor conductors. The tip is then scanned along the surface at a constant distance $s$ (to zero order) by means of a feedback control unit that maintains $I_{T}$ constant. As sketched in Fig. 1, both fine approach and scanning are effected by calibrated piezoceramic rods producing displacements of several $\AA / V$. The $z$-displacement (proportional to $V_{z}$ recorded along successive scans yields a nondestructive topographic profile (dotted line) provided the tip neither accidentally touches the surface nor induces local fields causing atoms to jump.

In contrast to experiments with solid (e.g. oxide) barriers, initiated by Giaever

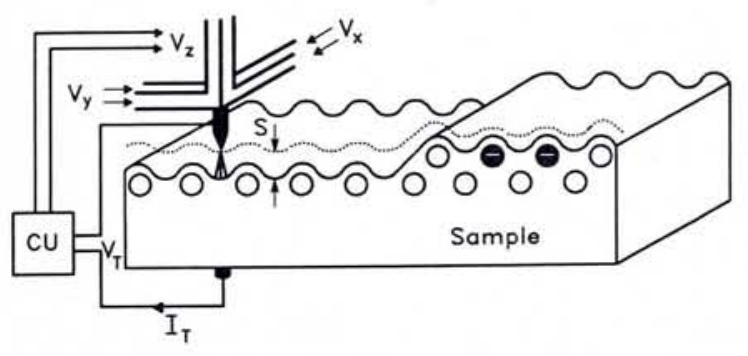

Fig. 1 - Principle of STM: The probing tip (black) is actuated by voltages independently applied to three mutually orthogonal piezodrives. While the tip is scanned along the sample by $V_{x}, V_{y}$, the control unit, $C U$, records and supplies the voltage $V_{z}$ required to keep the tunnelling current $I_{T}\left(V_{T}\right)$ constant.

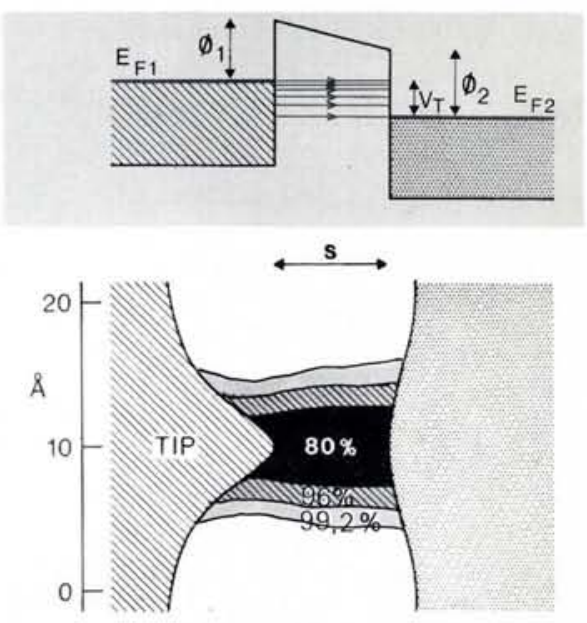

Fig. 2 - Distribution of tunnelling current density in energy (top) and space (bottom). (Ref. 3), Copyright 1986, IBM.

in 1960, the new technique relies on tunnelling through vacuum or an inert medium, like liquid $\mathrm{He}$, thus permitting in situ investigations of local surface changes induced by external means, or by the tip itself. Recently STM images of relatively inert surfaces like gold and graphite have been obtained in air and water ${ }^{3,4}$ ).

To obtain stable images, differing only by small drifts, required much ingenuity and perseverance. Mechanical vibrations and noise had to be suppressed. In the 1970's a few groups tried, but none could reach the goal of controlled approach, vacuum tunnelling and scanning. The specific designs discussed below all rely on an isolation and damping system against external disturbances and on a coarse approach mechanism that brings the sample within the working range (100 $\AA$ to several $\mu \mathrm{m})$ of the piezodrives. The influence of internal vibrations generated by the motion of the tip is avoided by setting the cutoff frequency of the feedback well below the lowest mechanical eigenfrequency of the tip-scanning unit.

Parameters determining the tunnelling current distribution, and hence the sen- 
sitivity and resolution of STM, are illustrated in Fig. 2. Assume for simplicity that the barrier is vacuum and that both sample and tip are good conductors. The applied voltage $V_{T}$ then appears across the separating potential barrier, and the height of the latter is approximately the average $\phi=\left(\phi_{1}+\phi_{2}\right) / 2$ of the tip and sample work functions. Its thickness $s$ is the distance between the turning points of electrons at a given energy within the window defined by the corresponding Fermi levels $E_{F 1}, E_{F 2}$ lassuming rapid equilibration within each electrode and negligible thermal excitation above the barrier). If $V_{T}<<\phi_{1}, \phi_{2}$, the distance dependence of $I_{T}$ is dominated by the exponential decay of the transmission coefficient at $E_{F 1} \approx E_{F 2}$. For free electrons tunnelling between parallel electrodes, the current density would be

$j \approx\left(e^{2} / h \pi\right)(\kappa / s) V_{T} \exp (-2 \kappa s)$, where $h / e^{2}=25.8 \mathrm{k} \Omega$ and

$2 \kappa\left(\AA^{-1}\right)=1.025 \sqrt{ }[\phi(e V)]$.

For a curved tip $I_{T}$ is approximately obtained by integrating (1) laterally about the closest separation $s$, thus giving an effective tunnelling area of $2 \sqrt{ }(r / \kappa)$ diameter for a tip of radius $r$ at its apex. This estimate of the lateral resolution $L$, e.g. $50 \AA$ for even a smooth tungsten field emission tip with $r=1000 \AA$ and $\phi=$ $4.5 \mathrm{eV}$, looked promising enough and motivated Binnig and Rohrer to build their first STM 5, 6). Their ability to observe monoatomic steps with apparent widths $<10 \AA$ led them to conjecture that the ground tungsten wires they used for tips supported small clusters of atoms. The extreme sensitivity of $I_{T}$ to $S$ naturally selects the "minitip" closest to the sample $\left.{ }^{6}\right)$ : the dream of resolving single surface atoms (schematically indicated by circles in Fig. 1) suddenly seemed within reach! Subsequent theories demonstrated that $L$ is of order $1.6 \sqrt{ }$ $[(r+s) / \kappa]$, but can also be smaller if, for instance, a host or a foreign atom with an outwards-directed orbital sits at the apex of the tip.

The two future laureates also proposed extensions of STM, namely local tunnelling spectroscopy and barrierheight profiling $\left.{ }^{6}\right)$. In the former one records the response $d l_{T} / d V_{T}$ to a small rapid modulation as $V_{T}$ is slowly swept to bring specific electronic states into energy coincidence with, for instance, the Fermi level of the tip. Such densityof-states effects modify the prefactor, but not the exponential in (1), at least for small $V_{T}$. A measurement of $d l_{T} / d s$ via modulation of $V_{z}$ therefore reveals local variations in $\phi$ due, e.g. to adsorbates (black circles in Fig. 1). Such variations cause changes in the apparent topogra-

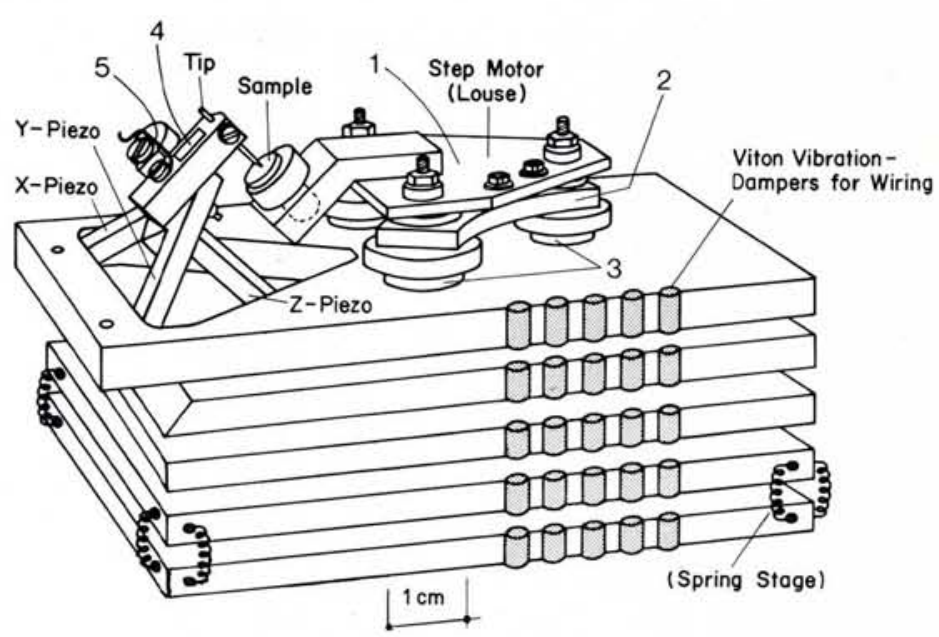

Fig. 3 - Schematic diagram of "pocket-size" STM. (Ref. 9), Copyright 1986, the American Institute of Physics.

phy that are difficult to recognize unless $\phi$ is simultaneously recorded.

\section{Experimental Aspects: Early Work}

The idea of STM arose in 1978 during a visit by Binnig to discuss research plans with Rohrer while he was writing up his doctoral dissertation. After settling in Zürich in the fall, Binnig started designing and testing various components with his uncanny ability to see the essential. He was helped by Rohrer's former experienced technician, Christoph Gerber, who has since then instructed hundreds of visitors and newcomers to the field. Binnig and Rohrer still pursued their previous research interests, but the situation changed in autumn 1981 when a working STM design took shape and an eager young technician, Edmund Weibel, joined the group.

Initially magnetic levitation above a superconducting lead bowl coated with aluminium provided vibration isolation and eddy-current damping. A linear decay of $\log I_{T}$ vs. $s$ was observed after a heuristic cleaning procedure, with $\phi$ as high as $3.2 \mathrm{eV}$ excluding tunnelling through an oxide or other contamination layer ${ }^{5}$ ). Preliminary STM scans of Au and Pt surfaces were shown together with that evidence at LT16 in August 1981. An expert in junction tunnelling sitting next to me exclaimed "my God, I have dreamed of doing this for the last twenty years!".

The second STM enabled careful surface preparation in UHV and revealed the above-mentioned narrow steps on $\mathrm{Au}(110)^{6}$ ) and, later, $\mathrm{Si}(111)$, as well as correlated topographic and $\phi$ scans for Au clusters on that surface ${ }^{7}$ ). Vibration isolation via a two-stage spring system with Viton connectors kept noise in $s$ below $0.2 \AA$. (This was reduced to 0.05 $\AA$ in the third STM $^{8}$ ) which also includ- ed LEED and Auger surface diagnostics.) As in later designs, including the compact one sketched in Fig. $3^{9}$ ), a "louse" consisting of a piezoplate (2) on three metal feet (3) separated from a ground plate by high dielectric constant insulators ensured coarse approach in steps of $100 \AA$ to $1 \mu \mathrm{m}$ via sequential clamping of the feet in harmony with contractions and elongations of the body (2). Better procedures for obtaining stable minitips in situ were developed, e.g. applying up to $100 \mathrm{~V}$ for a certain time.

At his first invited talk at the German Physical Society meeting in April 1982 Binnig claimed that $\simeq 3 \AA$ wide features occasionally appearing in adjacent scans were single adsorbates. Soon afterwards individual close-packed [001] atomic rows on the reconstructed Au(110) surface could be imaged. This observation prompted theorists to get to work and provided a basis for testing estimates of the resolution.

But it was the first STM image of the puzzling $7 \times 7$ reconstruction of annealed $\mathrm{Si}(111)$ that generated the most excitement. The features in the rhombohedral unit cell apparent in Fig. 4 (deep minima at the corners, 12 maxima associated with host adatoms, lower half on the [211] side ${ }^{10}$ ) was confirmed in differently doped samples ${ }^{11}$ ), and later by groups at AT\&T Bell Laboratories ${ }^{12}$ ), IBM Yorktown Heights ${ }^{3,4}$ ) and elsewhere.

\section{New Developments}

Rohrer received his first informal award for STM at a workshop in January 1983 for the relief shown in Fig. 4 (top) that was quickly assembled by cutting and glueing $x-y$ recorder traces on cardboard. Soon afterwards he energetically pushed computer-assisted STM data 

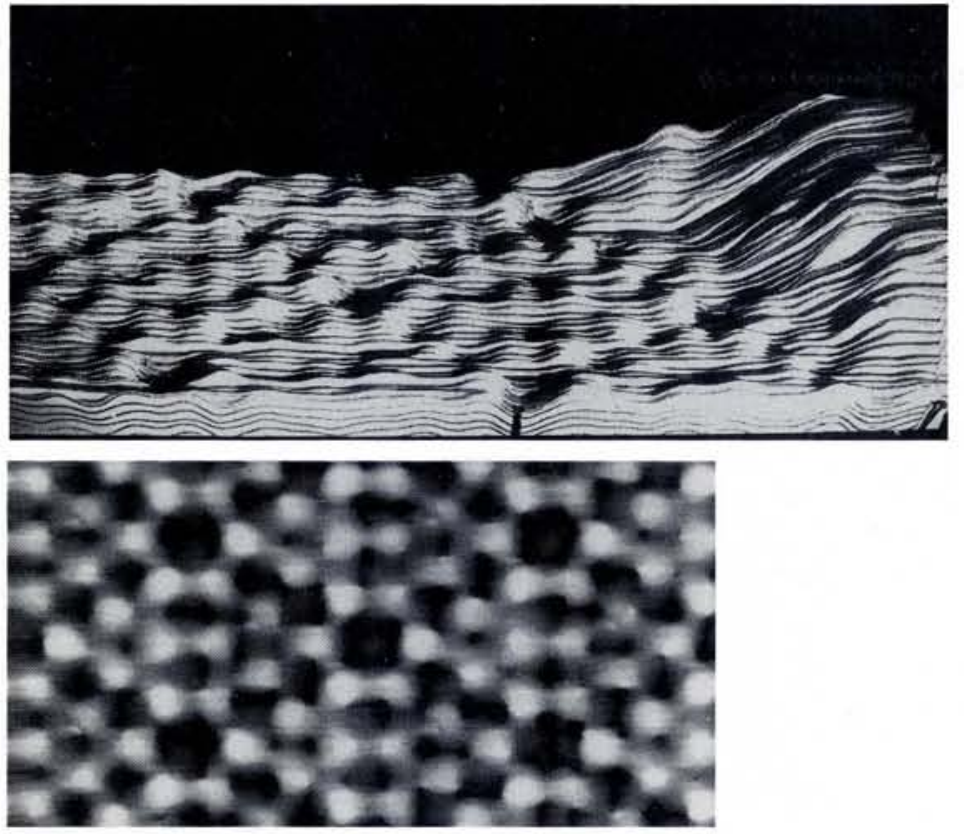

Fig. 4 - First STM image of $7 \times 7$ reconstructed Si(111) surface: original traces (top) and averaged grey-tone image (bottom); the closest-lying maxima are $6.65 \AA$ apart. (Ref. 10), Copyright 1983, the American Physical Society.

handling and image processing, as well as the application of STM to materials science, electrochemistry and biology via collaborative efforts. Some of us at the IBM Zürich Laboratory switched to STM-related research. Numerous visitors came by and profited from the experience already acquired, while other groups started building STM's on their own. Joint projects financed by IBM Europe and the institutions concerned were established with universities in Madrid (Autonoma), Marseilles (Luminy), Berne and Zürich (ETH). Sessions and symposia on STM started being regular features at scientific meetings.

The fruits of the first collaborations obtained with the third STM are summarized in several reviews ${ }^{118}$ ). They include observations of single adsorbed oxygen atoms on $\mathrm{Ni}(110)$, of the subtle large-scale reconstruction of $\mathrm{Au}(100)$, of the spiral structure of DNA and of the neck of a virus. Somewhat later local tunnelling spectroscopy confirmed the existence of both occupied and unoccupied states (depending on the sign of $V_{T}$ ) confined in front of or close to various surfaces, while scanning revealed that some of the latter were also localized laterally, e.g. where unsaturated bonds are expected between adatoms on $7 \times 7 \mathrm{Si}(111)$. These results were reported at a workshop in Oberlech in July 1985 where about twenty different groups or investigators working in STM reviewed their progress ${ }^{3}$ ). Subsequent $V_{T}$-dependent studies by two groups at IBM Yorktown Heights clarified the nature of such surface states for the
$7 \times 7$ and for the metastable $2 \times 1$ reconstruction of that surface. They were highlights at the first international conference on STM in Santiago de Compostella last summer ${ }^{4}$ ).

Other highlights originated from the atomic force microscope invented during Binnig's sabbatical at Stanford ${ }^{13}$ )

\section{T $\prod_{\text {ZÜRICH }}$}

RESEARCH POSITION in Experimental Particle Physics

At the Institute for Intermediate Energy Physics of the Swiss Federal Institute of Technology (ETH) in Zürich a postdoctoral research position is available.

The research connected with the present position is in the field of symmetry violations (P,T), interplay of weak and strong interactions, and the study of the few nucleon system with polarized particles. Most experiments are performed at the Swiss Institute for Nuclear Research (SIN),

Candidates should have $\mathrm{Ph}$.D. or equivalent research experience in particle or nuclear physics. They are expected to take an active part in the design, execution and analysis of the experiments. The duration of the contract is one to three years with an extension possible. The salary will be approx. Swiss Francs 50000.- to 60000 --, depending on experience and age.

Candidates should send a curriculum vitae and arrange for two letters of reference to be sent as soon as possible to:

Prof. J. Lang, Institut für Mittelenergiephysik, ETH-Zürich, CH - 8093 Zürich, Switzerland.

\section{Experimental Nuclear Physicist}

There is a vacancy for an experimental physicist to join the Nuclear Structure Division at Daresbury Laboratory, an establishment of the Science and Engineering Research Council which operates major national facilities for scientific research

The group is involved in carrying out and supporting a research programme on the Nuclear Structure Facility (NSF), a large tandem accelerator which is operating at up to $20 \mathrm{MV}$ on terminal

- The successful applicant will be required to organise and direct work necessary for the operation, maintenance and development of equipment used primarily in gamma-ray programmes aimed at studies of nuclear collective motion, nuclear reactions and nuclei far from stability. Candidates will be expected to carry out research in conjunction with university users on the NSF and to play an active role in initiating and developing new programmes. Other duties will involve the overall coordination and scheduling of the scientific programme and the assessment of future needs for instrumentation and other facilities on the N.S.F.

Applicants should have a good honours degree (or equivalent qualification) in physics and several years postgraduate experience in experimental nuclear research. $\mathrm{APh} . \mathrm{D}$ qualification in nuclear physics and a period of relevant post-doctoral experience would be an advantage.

The appointment will be made in the grade of Senior Scientific Officer on a salary scale of $£ 10,558$ to $£ 13,762$ per annum; the starting salary will depend on qualifications and experience. The superannuation scheme is non-contributory.

CLOSING DATE: Friday 13th February, 1987.

Application forms may be obtained quoting ref DL/974 from - The Personnel Officer,

Science and Engineering Research Council, Daresbury, Warrington WA4 4AD.

Telephone (0925) 603467.

Further information is

available from

Dr J S Lilley on

(0925) 603558 


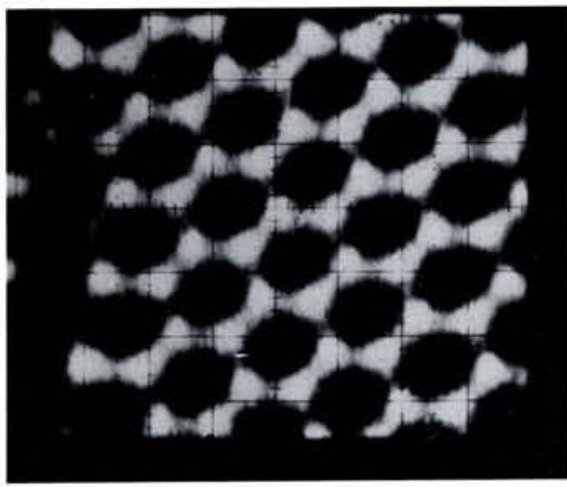

Fig. 5 - Real-time STM image of cleaved graphite. Minima are only $2.42 \AA$ apart.

and the atomic resolution studies of cleaved graphite performed before his departure with the STM shown in Fig. 3. A stack of stainless-steel plates separated by Viton dampers provided enough vibration isolation for stable operation in air. When placed into the UHV chamber of a scanning electron microscope, this compact instrument could produce traces of excellent clarity ${ }^{9}$ ) and the corresponding image clearly shows minima with the expected spacing. Although only every second surrounding carbon atom appears as a slightly shifted maximum in that picture, Binnig and coworkers very recently managed to image all six, albeit in a narrow range of current, as shown in Fig. 5. This photograph was taken in a few seconds direct from an oscilloscope driven by a fast-scanning STM operating in liquid $\mathrm{He}$ similar to one that was built in one day in Stanford ${ }^{4}$ ).

\section{REFERENCES}

1. Ruska E., The Early Development of Electron Lenses and Electron Microscopy (S Hirzel Verlag, Stuttgart) 1980.

2. Quate C.F., Phys. Today 39 (August 1986)

26; Salvan F., La Recherche 181 (1986) 1202.

3. IBM J. Res. Develop. 30 (1986) 355, 430

4. Proceeding of the STM'86 Conference,

Surf. Sci., to be published.

EPS Divisions, Sections and Group

Astronomy and Astrophysics Division Solar Physics Section

Atomic and Molecular Physics Division

Atomic Spectroscopy Section

Chemical Physics

Electronic and Atomic Collisions

Molecular Physics

Computational Physics Group

Condensed Matter Division

Liquids Section

Low Temperature Physics Section

Macromolecular Physics

Magnetism

Metal Physics

Semiconductors and Insulators

Surfaces and interfaces

High Energy \& Particle Physics Division

Interdiv. Group on Expt1. Phys. Control Systems

Interdiv. Group on Physics for Development

Interdiv. Group on Physics
Nuclear Physics Division

Nuclear Physics Din

Optics Division

Plasma Physics Division

Quantum Electronics Division

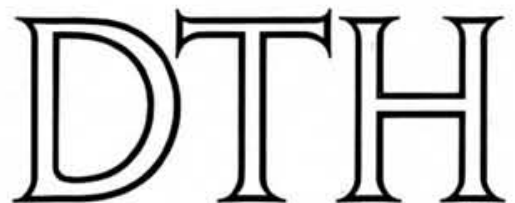

THE TECHNICAL UNIVERSITY OF DENMARK

\section{Associate Professor in Experimental Surface Physics}

At the Technical University of Denmark a new Surface Science Research Group is being created. When fully staffed it is planned to consist of 3-4 faculty positions. The first of these, at the associate professor (lecturer) level is now open at the Laboratory of Applied Physics II.

Applicants are expected to have several years of post doctoral research experience with surface spectroscopies, preferably also with some applications to industrial problems.

The applicant must be able to undertake teaching in elementary and advanced physics courses.

The successful applicant is expected to play a major role in the build up of the surface physics groups and the acquisition of equipment.

Further information can be obtained from Dr. Steen Mørup, Laboratory of Applied Physics II, Building 307, The Technical University of Denmark, DK-2800 Lyngby, Denmark. Tel. (45) (2) 882488 , ext. 2321.

Curriculum vitae including relevant publications, if possible in three copies, and an account of teaching experiences are to be directed to:

Faculty of Basic Sciences, Building 101

Technical University of Denmark,

DK-2800 Lyngby, Denmark

not later than 6 January, 1987.

5. Binnig G., Rohrer H., Gerber Ch. and Weibel E., Appl. Phys. Lett. 40 (1982) 178.

6. Binnig G., Rohrer H., Gerber Ch. and Weibel E., Phys. Rev. Lett. 49 (1982) 57.

7. Binnig G. and Rohrer H., Surf. Sci. 126 (1983) 236.

8. Binnig G. and Rohrer H., Sci. Am. 253 (August 1985) 50.

9. Gerber Ch., Binnig G., Fuchs H., Marti O. and Rohrer H., Rev. Sci. Instrum. 57 (1986) 221.

Europhysics News is the official journal of the European Physical Society which comprises 29 National Societies, Academies and Group, about 4000 Individual Members and 70 Associate Members. Governing boMembers and 70 Associate Members. Governing bo-
dies of EPS are the General Meeting, Council and an elected Executive Committee responsible for detailed policy. EPS promotes the collaboration of physicists throughout Europe, organising and harmonising conferences and publications, improving physics education, rences and publications, improving physics education, ships to sponsored schools in Erice. EPS publishes in addition to Europhys. News, Europhysics Letters lin partnership with national societies), European Journal of Physics (in partnership with The UK Inst of Phys I Europhys. Conf. Abs., Europhys. Ed. Nows. Individual Members receive Europhys. News free of charge (price to insts.: Sw.Fr. 90--/a), Europhys. Lett. at Sw.Fr. 70.-1a (insts. 595.-), rebates on many other publications and on conference fees. Annual EPS membership fee for Individual Members belonging to an EPS member society is: SwFr. 44 - indepent is: Sw.Fr. $44,-;$ independent members: Sw.Fr. 132,-;
10. Binnig G., Rohrer H., Gerber Ch. and Weibel E., Phys. Rev. Lett. 50 (1983) 120. 11. Binnig G. and Rohrer H., Physica 127B (1984) 37, and in Trends in Physics 1984 (J. Janta and J. Pantoflicek, eds. EPS) 1985, p. 38 .

12. Becker R.S., Golovchenko J., Higashi G.S. and Swartzentruber B.S., Phys. Rev. Lett. 57 (1986) 1020 and references therein. 13. Binnig G., Quate C.F. and Gerber Ch., Phys. Rev. Lett. 56 (1986) 930.
Editor: E.N. Shaw

Editorial Board:

K. Appert, A. Baratoff, F. James,

M. Lehmann, M. Mayor, J. Muller

Editorial and Advertising Office at the EPS Secretariat

Address: EUROPEAN PHYSICAL SOCIETY P. O. Box 69 CH- 1213 Petit-Lancy 2 Switzerland

Telephone: Geneva (22) 931130

Telex: 428024 eps ch

Cables: europhys genève

Printed by: Pfirter frères sa CH-1213 Petit-Lancy/Switzerland 